\title{
Random Perturbations of Bifurcation Diagrams
}

\author{
FRITZ COLONIUS \\ Institut für Mathematik. Universität Augsburg, 86159 Augsburg, Germany \\ WOLFGANG KLIEMANN \\ Department of Mathematics. Iowa State University. Ames, IA 5(1010, U.S.A.
}

\begin{abstract}
Random perturbations of one dimensional bifurcation diagrams can exhibit qualitative behavior that is quite different from that of the unperturbed, deterministic situation. For Markov solutions of one dimensional random differential equations with bounded ergodic diffusion processes as perturbations, effects like disappearance of stationary Markov solutions ('break through'), slowing down, bistability, and random symmetry breaking can occur. These effects are partially the results of local considerations, but as the perturbation range increases, global dynamics can alter the picture as well. The results are obtained via the analysis of stationary solutions of degenerate Markov diffusion processes, of stationary, non-Markovian solutions of stochastic flows, and of Lyapunov exponents of stochastic flows with respect to steady states.
\end{abstract}

Key words: Bifurcation, stationary Markov solutions, stationary flow solutions, control sets.

\section{Introduction}

Bifurcation theory of one dimensional deterministic vector fields depending on a parameter has led to a classification of bifurcation types for these systems, see, e.g., [13], [16], [22]. While random perturbations of dynamical systems are quite well understood (see, e.g., [17], [24]), various approaches have been suggested to capture the essence of stochastic bifurcation phenomena for the various bifurcation types. These approaches either work within the Markovian framework and look for changes in the form of invariant densities ([15], [25]), or use Lyapunov exponents of stationary stochastic flows (as the analogue of eigenvalues passing through the imaginary axis) ([1], [5]). Most of this work is in the context of stochastic differential equations, i.e., white noise perturbations.

In this paper, we consider random perturbations of one dimensional bifurcation diagrams with bounded, stationary noise, which comes from an underlying ergodic diffusion process on a compact manifold. We are interested in the Markov solutions of these systems, which basically means that the initial values are independent of the future of the noise. More precisely, consider a (deterministic) system $\dot{x}=X(x)+\alpha Y(x)$, where $\alpha \in \mathbb{R}$ is a bifurcation parameter. Let $\alpha_{t}^{\rho}$ be a stationary, ergodic process (coming from an underlying Markov diffusion) with values in $[\alpha-\rho$, $\alpha+\rho$ ] and consider the family $\dot{x}=X(x)+\alpha_{1}{ }^{p} Y(x), \rho \geq 0$, of random perturbations of the deterministic system. The problem is to describe the qualitative long term behavior of all Markov solutions of the stochastic systems, i.e., the stationary solutions, convergence towards these solutions, and certain features of the transients. This requires the use of two parameters, namely $\alpha$ and $\rho$, in order to obtain a complete picture. The model above is formulated using perturbations, 
of the bifurcation parameter $\alpha$ itself, but the results obtained here carry over to the case, where other parameters in the vector fields $X$ and/or $Y$ are subjected to bounded noise. Perturbations of the bifurcation parameter have the advantage that the behavior of the stochastic system can be visualized easily.

Our main results for one dimensional systems with small perturbation range are as follows. (In the sequel, $\alpha_{0}$ denotes the deterministic bifurcation point.)

In the saddle-node bifurcation, stationary Markov solutions exist for $\alpha \geq \alpha_{0}+\rho$. If $\alpha \in\left(\alpha_{0}-\rho\right.$, $\alpha_{0}+\rho$ ), the (transient) Markov solutions experience slow down, and for $\alpha \geq \alpha_{0}+\rho$ there also exist regions of bistability, i.e., areas of the state space from which two different stationary solutions can be reached with positive probability.

If two bifurcation branches intersect at $\alpha_{0}$, the behavior of the stochastic system depends on the sign of the slope of the branches at $\alpha_{0}$ : If the signs are different, stable branches turn into stationary Markov solutions, and unstable branches into areas of bistability. If the signs are equal, no stationary Markov solutions exist for $\alpha \in\left(\alpha_{0}-\rho, \alpha_{0}+\rho\right)$. The Markovian solutions will pass through the area given by these branches, but they will slow down considerably.

As the perturbation range $2 \rho$ increases, global dynamics may change the picture, e.g., stationary Markov solutions and/or regions of bistability may disappear, leading to new areas of transient behavior with considerable slow down.

These observations do not depend on the specific statistics of the random process $\alpha_{i}^{p}$, but only on the perturbation range. The shape of invariant densities, exit probabilities and exit times from bistability regions, and the amount of slow down, however, do depend on the statistics of $\alpha_{t}^{\rho}$. Our figures for these quantities present the essential behavior which can also be seen in simulations.

For the existing stationary Markov solutions we analyze their region of attraction, i.e., the sets of initial values for which the corresponding Markov solutions converge in distribution to the stationary one. If a bifurcation branch of the deterministic diagram is constant, i.e., horizontal around a bifurcation point, a different technique has to be used to analyze stability, because unstable common steady states are Markov stationary solutions. In this case we compute the Lyapunov exponents of the stochastic system with respect to the corresponding steady state to determine, at which point the steady state Markov solution changes its stability behavior. Away from the common steady state, techniques as described above can be used. For related white noise results we refer to [1] and [3].

The goal of this paper is to understand the behavior of Markov solutions of $\dot{x}=X(x)+$ $\alpha_{t}^{p} Y(x)$, where $\alpha_{?}^{p}$ comes from an underlying ergodic Markov diffusion process. It turns out, however, that this cannot be achieved by looking only at stationary Markov solutions. Effects like bistability or slowing down are intimately related to the existence of non-Markovian stationary solutions, so called stationary flow solutions. In fact, including these solutions leads to complete stochastic bifurcation diagrams depending on the parameters $\alpha$ and $\rho$, because branches of stationary Markov solutions can be continued via stationary flow solutions for $\alpha$-values, where stationary Markov solutions may not exist. In this sense, our work is complementary to [1].

This paper is organized in the following fashion: Section 2 presents the mathematical background material on existence, uniqueness, and stability of stationary (Markov and flow) solutions. Associated control systems and their perturbation theory (over the entire time axis $\mathbb{R}$ ) play an important role. Furthermore, Lyapunov exponents are introduced and computed for stochastic flows with respect to steady states. In Section 3 random perturbations of bifurcation diagrams without constant (i.e., horizontal) bifurcation branches are discussed. Section 4 contains the singular case of diagrams with constant bifurcation branches. A comparison shows that 
diagrams with horizontal branches are in some sense the limiting case between intersections of bifurcation branches with equal sign of the slope, and those with different sign of the slopes. resulting in completely different behavior of the stochastic systems, as described above. The implications for modeling of systems with random uncertainties are obvious.

Sections 3 and 4 give a complete overview over perturbations of one dimensional bifurcation diagrams with bounded noise (except for one case, which we could not solve using the techniques with Section 2, see 4.1 with $\alpha \in\left(\alpha_{0}, \alpha_{0}+\rho\right)$ ). Since we consider perturbations of the bifurcation parameter $\alpha$, the results bear a certain resemblance to (deterministic) dynamic bifurcation theory, compare [4]. Background material for the analysis of perturbed Hopf bifurcations appears in [6].

\section{List of symbols}

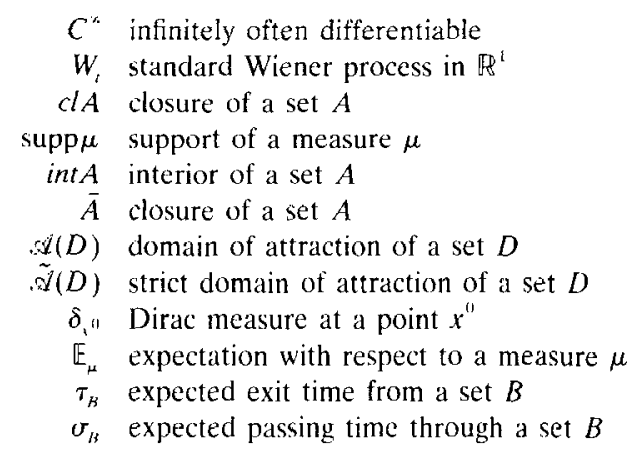

\section{Stochastic Systems and Their Stationary Solutions}

This section presents some background material on existence, uniqueness, and stability of stationary solutions of stochastic systems. We restrict ourselves here to systems with one dimensional state space (the real line $\mathbb{R}$ ) and randomly perturbed bifurcation parameter $\alpha \in \mathbb{R}$. Perturbations of other system parameters lead to the same kind of results, but are more difficult to visualize.

Consider a nonlinear (deterministic) system of the form

$$
\dot{x}=X(x)+\alpha Y(x), x_{0}=x(0) \in \mathbb{R} .
$$

where $\alpha$ is the bifurcation parameter. We assume throughout this paper that $X$ and $Y$ are $C^{*}$ vectorfields, such that $X+\alpha Y$ has at most finitely many zeros for all $\alpha \in \mathbb{R}$. In order to avoid problems of explosion in finite time, we assume that the vector fields are Lipschitz - otherwise one would have to use local solutions. Random perturbation of $\alpha$ via a bounded Markov diffusion process leads to the following stochastic set up:

$$
\dot{x}=X(x)+\alpha_{i} Y(x),
$$

where we assume the following model for the stochastic process $\alpha_{t}$ :

A background noise $\beta_{t}$ is given by the stochastic differential equation

$$
\mathrm{d} \beta_{t}=Z_{t)}\left(\beta_{t}\right) \mathrm{d} t+Z_{1}\left(\beta_{t}\right) \circ \mathrm{d} W_{t}
$$


on a compact, one dimensional manifold $M$ (which can be taken without loss of generality to be the circle $S^{1}$ in $\mathbb{R}^{2}$ ), with $C^{\infty}$ vectorfields $Z_{0}$ and $Z_{1}$. ("。" denotes the symmetric or Stratonovic stochastic differential.) We assume that $Z_{1}(p) \neq 0$ for all $p \in M$, and then equation (B) has a unique stationary, ergodic solution, denoted by $\beta_{t}$, with invariant density called $\eta$. The background noise $\beta$, influences the system via a family of $C^{x}$ subjective maps

$$
f^{\rho}: M \rightarrow[\alpha-\rho, \alpha+\rho], \rho \geq 0,
$$

where $\rho$ determines the size of the perturbation $\alpha_{t}^{\rho}:=f^{\rho}\left(\beta_{t}\right)$. Note that for each $\rho \geq 0, \alpha_{t}^{\rho}$ is itself a stationary process, whose invariant density is $f^{\rho}(\eta)$. The pair process $\left(\beta_{t}, x_{t}\right)$ is governed by the two dimensional stochastic differential equation $(B, S)$.

In order to analyze the behavior of the stochastic system (S), we are looking for stationary solutions $x_{i}$ such that $\left(\beta_{t}, x_{t}\right)$ is a stationary pair. It turns out that stationary Markov solutions of the $\operatorname{SDE}(\mathrm{B}, \mathrm{S})$ are not sufficient to describe the phenomena of random perturbations in bifurcation diagrams, one needs the concept of stationary flow solutions, which we introduce next.

Let $\Omega^{\rho}=C(\mathbb{R},[\alpha-\rho, \alpha+\rho])$ be the space of continuous functions with values in $U^{\rho}:=[\alpha-$ $\rho, \alpha+\rho]$, i.e., the trajectory space of $\alpha_{t}^{\rho}$. For each $\rho \geq 0$ the stationary process $\alpha_{t}^{\rho}$ induces a measure $P^{\rho}$ on $\Omega^{\rho}$, which describes for a measurable set of functions the probability that this set is realized as trajectories of $\alpha_{t}^{\rho}$, see e.g., [21]. On $\Omega^{\rho}$ we define the shift operator $\Theta$ by

$$
\Theta: \mathbb{R} \times \Omega^{\rho} \rightarrow \Omega^{\rho}, \Theta(t, \omega(\cdot))=\omega(t+\cdot) .
$$

In this set up, $P^{\rho}$ is $\Theta$-invariant and ergodic for each $\rho \geq 0$. The solutions of (S) are now described as a random flow $\Phi^{\rho}$ in the following way:

$$
\begin{aligned}
& \Phi^{\rho}: \mathbb{R} \times \Omega^{\rho} \times \mathbb{R} \rightarrow \Omega^{\rho} \times \mathbb{R}, \\
& \Phi_{l}^{\rho}(\omega, x)=(\Theta(t, \omega), \varphi(t, x, \omega)) \text { for } t \in \mathbb{R},
\end{aligned}
$$

where $\varphi(t, x, \omega)$ denotes the solution of (S) at time $t$ for the random trajectory $\omega$ with initial value $\varphi(0, x, \omega)=x \in \mathbb{R}$.

We are now ready to define stationary solutions of the random system $(\mathrm{S})$ :

1. DEFINITION. A stochastic process $x^{\rho}$ is a solution of $(\mathrm{S})$, if the family of trajectories $x^{\rho}(\cdot, x, \omega): \mathbb{R} \rightarrow \mathbb{R}$ satisfies the differential equation $P^{\rho}$-almost surely, with $x^{\rho}(0, x, \omega)=x(\omega)$. Here $x(\omega)$ is a random variable which is measurable with respect to the canonical $\sigma$-algebra on $\Omega^{\rho}$, i.e., $x(\omega)$ need not be independent of the future of $\alpha^{\rho}$. A solution $x^{\rho}$ of (S) is a stationary flow solution (of (1)), if the joint distribution $Q^{\rho}$ of $\left(\Theta, x^{\rho}\right)$ on $\Omega^{\rho} \times \mathbb{R}$ (with marginal $P^{\rho}$ on $\Omega^{\rho}$ ) is invariant under $\Phi_{1}^{\rho}$ for all $t \in \mathbb{R}$. A solution $x^{\rho}$ of $(\mathrm{S})$ is a stationary Markov solution (of the SDE $(\mathrm{B}, \mathrm{S}))$, if $\left(\beta_{t}, x_{t}\right)$ is a stationary Markov process.

2. REMARK. All stationary Markov solutions lead to stationary flow solutions (see e.g., [11]), but the converse is not necessarily true (see Theorem 4). The difference lies in the problem of finding an initial random variable that is independent of the Wiener process $W_{t}$. The marginal of a stationary Markov solution on $M$ is, by construction, given by the invariant density $\eta$ of $\beta_{t}$, while the marginal of a stationary flow solution on $\Omega^{\rho}$ is $P^{\rho}$. Note that the projection of $P^{\rho}$ onto $U^{\rho}=f^{\rho}[M]$ is given by $f^{\rho}(\eta)$. By Hörmander's theorem (see, e.g. [14]) the stationary Markov solutions $\left(\beta_{t}, x_{t}^{p}\right)$ have $C^{x}$ densities, if $x_{t}^{p}$ is not a single point. Otherwise, the invariant distribution is $\eta \times \delta_{x^{\prime \prime}}$, where $x_{t}^{\rho} \equiv x^{0}$. 
Although the state space of the stochastic system $(S)$ is one-dimensional, stationary solutions cannot be found via Feller's boundary classification (see, e.g. [12]), because the random process $\alpha$, is not white, i.e., (S) by itself is not an SDE. However, connections with the theory of nonlinear control systems allow us to find both kinds of stationary solutions quite easily. We begin with a discussion of stationary Markov solutions.

Associate with the stochastic system (S) the nonlinear control system

$$
\dot{x}=X(x)+u(t) Y(x), \quad x_{0} \in \mathbb{R}
$$

where $u(t) \in U^{\rho}$ and $U^{\rho}:=\left\{u: \mathbb{R} \rightarrow U^{\rho}\right.$, measurable $\}$ is the space of admissible control functions. For the following control theoretic concepts see, e.g. [7]. A set $D \subset \mathbb{R}$ is a control set of (C), if $D \subset c l\left\{y \in \mathbb{R}\right.$; there exist $u \in U^{\rho}$ and $t>0$ with $\left.\varphi(t, x, u)=y\right\}$, and if $D$ is maximal (with respect to set inclusion) with this property. Here " $c l$ " denotes the closure of a set, and $\varphi(t, x, u)$ is the solution of $(\mathrm{C})$ corresponding to the control function $u$, with initial value $x=\varphi(0, x, u)$. We denote the control sets of $(\mathrm{C})$ by $D_{i}, i=1, \ldots, k<\infty$. (Note that the number of control sets is finite, because $X+u Y$ has at most finitely many zeros.) The control sets are (partially) ordered by the relation $<$, defined as

$$
D_{i}<D_{j} \text { if there exists } x \in D_{i} \text { with } \Theta^{+}(x) \cap D_{j} \neq \emptyset .
$$

Here $\mathcal{O}^{+}(x)=\left\{y \in \mathbb{R}\right.$; there is $u \in \mathcal{U}^{\rho}$ with $\varphi(t, x, u)=y$ for some $\left.t \geq 0\right\}$ is the positive orbit of the point $x$. Open control sets are minimal, and closed control sets are maximal elements with respect to the order <. If a boundary point $y$ of some control set $D$ is not contained in $D$, then $y$ is called an exit point, otherwise it is an entrance point. Obviously, open control sets have two exit points, while closed sets have two entrance points. In particular, closed control sets $D$ are invariant, i.e., $\mathscr{O}^{+}(x) \subset D$ for all $x \in D$.

Concerning stationary Markov solution we have the following result, see [18]:

3. THEOREM. (i) Let $\left(\beta_{r}, x_{t}\right)$ be a stationary, ergodic Markov solution of (S) with invariant density $\mu$. Then there exists a (unique) invariant control set of (C) with supp $\mu=M \times \bar{D}$. Furthermore, ergodic Markov solutions are unique on invariant control sets, and all stationary Markov solutions are convex combinations of the ergodic solutions on the (finitely many) invariant control sets.

(ii) If $D \subset \mathbb{R}$ is a compact, invariant control set of $(\mathrm{C})$, then there exists a (unique) stationary, ergodic Markov solution on $M \times D$.

The situation for stationary flow solutions is somewhat more complicated. Obviously, there cannot exist any stationary solutions outside of control sets. On control sets of (C) we have the following result:

4. THEOREM. Let $D \subset \mathbb{R}$ be a control set of $(\mathrm{C})$ and suppose that $\cup\{D ; D$ is a control set $\}$ is bounded.

(1) Assume that no point in $\bar{D}$. the closure of $D$, is a zero of $X+u Y$ for all $u \in U$.

(i) If $D$ is invariant, then there exists (at least) one stationary flow solution in $D$.

(ii) If $D$ is a variant control set with $V:=\{u \in U ; X(x)+u Y(x) \neq 0$ for all $x \in \bar{D}\}=\emptyset$, then there exists a stationary flow solution in $\bar{D}$.

(iii) If $D$ is a variant control set with $V \neq \emptyset$, then no stationary flow solution exists in $\bar{D}$. 
(2) Assume that a boundary point $x^{0}$ of $D$ satisfies $X\left(x^{0}\right)+u Y\left(x^{0}\right)=0$ for all $u \in U$. Then there exists a stationary flow solution in $\bar{D}$.

Proof. The joint distribution $Q$ of a stationary flow solution $(\Theta, x)$ on $\Omega \times \mathbb{R}$ has marginal $P$ on $\Omega$, hence $Q$ can be disintegrated as $Q(\mathrm{~d} \omega, \mathrm{d} x)=\nu_{\omega}(\mathrm{d} x) P(\mathrm{~d} \omega) . Q$ is $\Phi$-invariant iff for $P$-almost all $\omega$ we have $\nu_{\omega}=\nu_{\Theta,(\omega)}$ for all $t \in \mathbb{R}$.

For a control set $D$ of $(\mathrm{C})$ and for $\omega \in \Omega$ define $D_{\omega}^{+}=\{x \in \bar{D} ; \varphi(t, x, \omega) \in \bar{D}$ for all $t \geq 0\}$. Now the problem of finding a stationary flow solution in $\bar{D}$ boils down to showing that for $P$-almost all $\omega$ one has $D_{\omega}^{+} \neq \emptyset$, see [7], Theorem 4.4 .

(1) (i) Under the assumption, the invariant control set $D$ is compact, and by invariance we have that $D_{\omega}^{+}=D$ for all $\omega \in \Omega$.

(1) (ii) In this case int $D \neq \emptyset$, and hence a stationary flow solution exists in $\bar{D}$ by [7], Proposition 4.8. Furthermore, the construction in the proof of [7], Proposition 4.8 yields: If there are two control sets $D_{1}$ and $D_{2}$, both of which satisfy the assumption of (1)(ii), with $\bar{D}_{1} \cap \bar{D}_{2} \neq \emptyset$, then there exist different stationary flow solutions in $\bar{D}_{1}$ and in $\bar{D}_{2}$.

(1) (iii) Since $\beta_{t}$ is a nondegenerate diffusion with $Z_{1}(p) \neq 0$ for all $p \in M$, and since $f^{\rho}$ is surjective, we have supp $P^{\rho}=\Omega^{\rho}$ for all $\rho>0$, see [20]. The vectorfields $X$ and $Y$ are continuous, and therefore $V \neq \emptyset$ means int $V \neq \emptyset$. Take $v \in$ int $V$, then either $X(x)+$ $v Y(x)<0$ or $>0$ for all $x \in \bar{D}$. Consider without loss of generality the first case. There exists an $\varepsilon>0$ such that $X(x)+v Y(x)<-\varepsilon<0$ for all $x \in \bar{D}$. Define $t_{v}:=\max \{t \geq 0$; $\varphi(t, x, v)=\inf D$ for some $x \in \bar{D}\}$. By the above $t_{v}<\infty$. Then there exists a neighborhood $N(v) \subset V$ such that $\varphi\left(t_{v}+1, x, \omega\right) \not \bar{D}$ for all $\omega \in \mathcal{N}(v):=\left\{\omega \in \Omega^{\rho} ; \omega(t) \in N(v)\right.$ for $\left.0 \leq t \leq t_{v}+1\right\}$. By nondegeneracy of $\beta_{t}$, the set $\mathcal{N}(v)$ has positive $P^{\rho}$-measure, but $D_{\omega}^{+}=\emptyset$ for all $\omega \in \mathcal{N}(v)$. Hence no stationary flow solution exists in $\bar{D}$ for the range $U$. Of course, a stationary flow solution does exist, when the range is restricted to $U \backslash V$.

(2) Obviously the solution $x^{0}$ is a stationary flow solution in $\bar{D}$. For the structure of the process in $D$ see the following corollary.

The following corollary describes the structures of the solution in control sets whose boundary contains common fixed points.

5. COROLLARY. Under the assumptions of Theorem 4(2) let $D$ be a control set with int $D \neq \emptyset$. Then there exists a subset $\hat{\Omega} \subset \Omega$ with $P \hat{Q}=1$, such that for a dense set in the weak ${ }^{*}$ topology in $\hat{\Omega}$ we have $D_{t \rightarrow 3}^{+} \cap D \neq \emptyset$.

Proof. We continue to use the notation from Theorem 4, and its proof. Denote $\hat{V}:=\{u \in U$; $X(x)+u Y(x) \neq 0$ for all $x \in D\}$, and $\hat{\mathcal{N}}=\{\omega \in \Omega ; \omega(t) \in \hat{V}$ for all $t \geq 0\}$. Since $\beta_{t}$ is an ergodic process, $P^{\rho \hat{V}}=0$. The periodic trajectories are dense in $\hat{\Omega}:=\Omega \backslash \mathscr{V}$ (see [9]), and by the construction in the proof of [7], Proposition 4.8(ii) we have for the periodic $\omega$ 's that $D_{\omega}^{+} \cap D \neq \emptyset$. It is not clear, however, whether this idea can be extended to set of full $P^{\rho}$-measure, since the control set $D$ under the assumptions of this corollary is not isolated.

6. REMARK. Uniqueness of stationary flow solutions in a control set $D$ cannot be guaranteed, in general. This depends on the number of fixed points of the right hand side of (C) for $u \in U^{p}$, compare the discussion in Section 3. The stability of the fixed points in $D$ also determines, how fast a Markov solution of $(S)$ crosses through $D$, i.e., whether effects of 'slowing down' can be observed in the vicinity of bifurcation points of (D). Again, we refer to Section 3 for more details. 
Theorems 3 and 4 underline the importance of control sets for the analysis of randomly perturbed bifurcation diagrams corresponding to (D). Reference [7] contains a complete description of control sets for systems with one dimensional state space. The next theorem describes, how the control sets of (C) are related to the underlying deterministic system (D), for varying control range $U^{\rho}, \rho \geq 0$.

7. THEOREM. Consider the deterministic system (D) and the control system $\left(\mathrm{C}^{\mu}\right)$ with control range $U^{\rho}, \rho>0$, both with $\alpha \in \mathbb{R}$ fixed.

(i) If $x^{\prime \prime} \in \mathbb{R}$ is a fixed point of $(D)$, then there exists, for each $\rho>0$, a control set $D^{\rho}$ of $\left(C^{\rho}\right)$ with $x^{\prime \prime} \in D^{p}$. Vice versa, if $x \in \mathbb{B}$ is not a fixed point of $(\mathrm{D})$ then there is $\rho^{\prime \prime}>0$ such that $x^{\prime \prime}$ is not contained in any control set of $\left(C^{p}\right)$ for $0<\rho<\rho^{0}$.

(ii) If $x^{\prime \prime} \in \mathbb{R}$ is a fixed point of $(D)$ with $X\left(x^{\prime \prime}\right)+u Y\left(x^{\prime \prime}\right)=0$ for all $u \in U^{p}$, then $\{x)$ is an (invariant) control set of $\left(\mathrm{C}^{p}\right)$.

(iii) Let $x^{\prime \prime} \in \mathbb{R}$ be a fixed point of (D) such that there exist $u_{1}, u_{2} \in U^{p}$ with $X\left(x^{n}\right)+u_{1} Y\left(x^{n}\right)<0$ and $X\left(x^{10}\right)+u_{2} Y\left(x^{0}\right)>0$.

(a) If $x^{0}$ is stable for (D), then $x^{0} \in$ int $C^{\mu}$, the interior of some invariant control set $C^{\rho}$ of $\left(\mathrm{C}^{\prime}\right)$ for $\rho>0$ small enough,

(b) if $x^{\prime \prime}$ is unstable for (D), then $x^{\prime \prime} \in$ int $D^{\rho}$, the interior of some variant control set $D^{\rho}$ of $\left(C^{\rho}\right)$ for $\rho>0$ small enough.

Proof. Note first of all that the cases (i)-(iii, b) are the only ones possible, because of the form of the control system $(\mathrm{C})$. The proof now follows from the direct construction of control sets in [7], or from [8], Corollary 5.3, by noticing that under the conditions in (iii) the pair $\left(0, x^{0}\right)$ is an inner point.

Theorem 7 says that for $\rho>0$ small the control sets of $\left(\mathrm{C}^{\rho}\right)$ develop around the fixed points of (D), and the control sets are variant or invariant depending on whether the fixed point is unstable, or stable. As $\rho$ increases, the control sets may increase and several controls sets may merge as the result of the global dynamics of $\left(\mathrm{C}^{\rho}\right)$. In any case, there are at most $n$ control sets of $\left(\mathrm{C}^{\rho}\right)$, where $n$ the maximal number of fixed points of $X+u Y$, as $u$ varies over $U^{p}$.

8. REMARK. As the range of the random perturbation $U^{\mu}=[\alpha-\rho, \alpha+\rho]$ increases (for $\alpha \in \mathbb{R}$ fixed), the number and the form of the control sets of $\left(\mathrm{C}^{\rho}\right)$ depend more and more on the global dynamics of $\left(\mathrm{C}^{p}\right)$, not just on the local properties of the fixed points of $(D)$, as is the case for small $\rho$. Hence random perturbations of bifurcation diagrams associated with (D) should be considered as depending on two parameters, namely $\alpha$ and $\rho$. This will be our point of view in the next sections.

Finally, we turn to stability of the stationary solutions of (S). Since we are interested in the Markovian behavior of (S), we consider here only stationary Markov solutions. Two cases have to be distinguished:

(I) The stationary Markov solution $\left(\beta_{t}, x_{t}\right)$ has an invariant density $\mu$ with supp $\mu=M \times \bar{D}$, where $D$ is an invariant control set of $(C)$ with int $D \neq \emptyset$. Define the domain of attraction $\mathscr{A}(D):=\left\{y \in \mathbb{R} ; \mathscr{O}^{+}(y) \cap D \neq \emptyset\right\}$ and the strict domain of attraction $\tilde{A}(D):=\{y \in \mathscr{A}(D)$; for $P^{\rho}$-almost all $\omega \in \Omega^{\rho}$ there exists $t_{\omega}$ such that $\left.\varphi\left(t_{\omega}, y, \omega\right) \in D\right\}$. By the support theorem (see [23] or [19]) and ergodicity of $\left(\beta_{t}, x_{t}\right)$ in $M \times D$, we know: The solution $x_{t}(y)$ of (S) with initial value $y \in \tilde{A}(D)$ converges in distribution towards the marginal of $\mu$ on $D$ (cp. [18] or [2]). (For 
$y \in \mathscr{A}(D)$ there exists a number $p, 0<p \leq 1$, and a set $\Lambda \subset \Omega^{\rho}$ with $P^{\rho}(\Lambda)=p$, such that the trajectories of (S) with $\omega \in \Lambda$ converge weakly towards the marginal of $\mu$ on $D$.) Note that $\mathscr{A}(D)$ or $\tilde{A}(D)$ can contain variant control sets of $(C)$. In this sense, $\tilde{A}(D)$ describes the (weak) stability domain of the stationary Markov solution $\left(\beta_{t}, x_{t}\right)$.

(II) The stationary Markov solution $\left(\beta_{t}, x_{t}\right)$ has invariant density $\mu=\eta \times \delta_{x^{0}}$, i.e., $x^{0}$ is a fixed point of $X+u Y$ for all $u \in U^{\rho}$. In this case the corresponding invariant control set is $M \times\left\{x^{0}\right\}$ with domain of attraction $\mathscr{A}\left(\left\{x^{0}\right\}\right)=\left\{x^{0}\right\}$. To overcome this difficulty, we use the concept of Lyapunov exponents to describe (almost sure) stability of $\left(\beta_{i}, x^{0}\right)$, which is the appropriate concept in this case (cp. [3] and Section 4). In order to obtain a formula for the Lyapunov exponent of the stationary solution $x_{t} \equiv x^{0}$, we linearize the system (S) around $x^{0}$ :

$$
\dot{v}=A v+\alpha_{t}(\omega) B v
$$

where $A=D_{x} X\left(x^{0}\right)$ and $B=D_{x} Y\left(x^{0}\right)$. The Lyapunov exponents of (2) are

$$
\lambda\left(\omega, v_{0}\right)=\limsup _{t \rightarrow \infty} \frac{1}{t} \log \left|v\left(t, v_{0}, \omega\right)\right|=A+B \cdot \limsup _{t \rightarrow \infty} \int_{0}^{t} \alpha_{\tau}(\omega) \mathrm{d} \tau,
$$

where $v\left(t, v_{0}, \omega\right)$ is the solution (2) with initial value $v\left(0, v_{0}, \omega\right)=v_{0}$. Since $\alpha_{1}$ is a stationary, ergodic process, we obtain

$$
\lambda^{\rho}\left(\omega, v_{0}\right)=\lambda^{\rho}=A+B \mathbb{E}_{\eta} f^{\rho}(p) \quad P^{\rho} \text {-almost surely for all } v_{0} \neq 0 .
$$

In particular, if $\alpha_{t}^{\rho}=f^{\rho}\left(\beta_{t}\right)$ has a symmetric invariant distribution in $U^{\rho}=[\alpha-\rho, \alpha+\rho]$, then $\lambda^{\rho}(\alpha)=A+B \alpha$, which is in this case independent of $\rho>0$.

If the Lyapunov exponent $\lambda$ is negative, then for $P^{\rho}$-almost all $\omega \in \Omega^{\rho}$ there exists an open neighborhood $N\left(x^{0}, \omega\right)$ of the stationary point $x^{0}$, such that the trajectories $x_{i}(y, \omega)$ of (S) converge towards $x^{0}$ exponentially fast for all $y \in N\left(x^{0}, \omega\right)$. In this sense, the collection $\left\{N\left(x^{0}, \omega\right)\right.$, $\left.\omega \in \Omega^{\rho}\right\}$ describes the (exponential) stability domain of the stationary solution $x_{t} \equiv x^{0}$. If $\lambda>0$, then solutions diverge from $x^{0}$ exponentially fast. These observations have led to the formulation of a stochastic bifurcation theory based on Lyapunov exponents, compare, e.g. [1].

The next result concerns the nonexistence of stationary Markov solutions in invariant control sets with adjacent stable fixed point.

9. PROPOSITION. Let $D \subset \mathbb{R}$ be an invariant control set of $(C)$ with int $D \neq \emptyset$. Suppose there exists $x^{10} \in \bar{D}$ such that $X\left(x^{10}\right)+u Y\left(x^{0}\right)=0$ for all $u \in U$. Assume that the Lyapunov exponent of (S) at $x^{\prime \prime}$ is negative. Then there exists no stationary Markov solution in $D$.

Proof. If $\lambda<0$, then there exists for all $\delta>0$ a neighborhood $N\left(x^{0}, \varepsilon\right):=\left\{x \in \mathbb{R} ;\left|x-x^{0}\right|<\varepsilon\right\}$ (with $\varepsilon>0$ arbitrarily small), a time $T>0$ and a constant $c>0$ such that $p:=P\{\omega \in \Omega$; $\left|\varphi(t, x, \omega)-x^{0}\right| \leq c e^{t \gamma}$ for all $\left.t \geq T, x \in N\left(x^{0}, \varepsilon\right)\right\}>1-\delta$ for some $\gamma \in(\lambda, 0)$. In particular, there is a time $\hat{T} \geq T$ such that $\int_{\hat{T}}^{x} P\left(t, x, N\left(x^{(1}, \varepsilon\right)\right) \mathrm{d} t>1-\delta$ for all $x \in N\left(x^{0}, \varepsilon\right)$, where $P(\cdot, \cdot, \cdot)$ denotes the $x$-component of the transition probability.

If there exists a stationary Markov solution $\left(\beta_{t}, x_{t}\right)$ of the $\operatorname{SDE}(\mathrm{B}, \mathrm{S})$ in $D$, then this solution has an invariant density $\mu$ with supp $\mu=M \times \bar{D}$ and $\mu(M \times D)=1$. Its marginal $\mu_{D}$ on $D$ satisfies $\operatorname{supp} \mu_{D}=\bar{D}$ and $\mu_{D}(D)=1$. Choose $\delta \in\left(0, \frac{1}{3}\right)$ and $\varepsilon>0$ according to the remarks above so that 
$\mu_{D}\left(N\left(x^{\prime \prime}, \varepsilon\right)\right)<\delta$. Denote by $\tau_{f}(\omega):=\inf \left\{t \geq \hat{T} ; x_{t}(\omega) \in N\left(x^{\prime \prime}, \varepsilon\right)\right\}$ the first hitting time of $N\left(x^{0}, \varepsilon\right)$ after $\hat{T}$. Since $\left(\beta_{t}, x_{t}\right)$ is ergodic, $\tau_{\varepsilon}<\infty P$-almost surely, hence there exists $\tau \geq \hat{T}$ with $P\left\{\tau_{f}(\omega) \leq\right.$ $\tau\}>1-\delta$. Therefore we obtain for $t \geq \tau+\hat{T}$

$$
\begin{aligned}
\mu_{D}\left(D \backslash N\left(x^{\prime \prime}, \varepsilon\right)\right)= & \int P\left(t, x, D \backslash\left(x^{0}, \varepsilon\right)\right) \mu(\mathrm{d} x) \\
= & \int_{N\left(x^{\prime \prime}, x\right\}} P\left(t, x, D W\left(x^{\prime \prime}, \varepsilon\right)\right) \mu_{D}(\mathrm{~d} x) \\
& +\int_{D \backslash N\left(x^{\prime \prime}, \varepsilon\right)} P\left(t, x, D W\left(x^{\prime \prime}, \varepsilon\right)\right) \mu_{D}(\mathrm{~d} x) \\
& <\delta+(1-\delta) \cdot \delta<1-\delta<\mu_{D}\left(D W\left(x^{\prime \prime}, \varepsilon\right)\right),
\end{aligned}
$$

which is a contradiction.

Vice versa, we expect that positivity of the Lyapunov exponent at $x^{11}$ implies the existence of a stationary Markov solution in $D$, just as in the white noise case, see [3]. However, the techniques from [3] do not apply to the bounded, colored noise case.

\section{Random Perturbations of Bifurcation Diagrams I: Regular Systems}

In this section we consider regular systems, i.e., stochastic systems of the form

$$
\dot{x}=X(x)+\alpha_{l}^{\rho} Y(x), \alpha,(\omega) \in U^{\rho}=[\alpha-\rho, \alpha+\rho]
$$

as described in Section 2, with the additional property that there is no common zero for all $X+u Y, u \in U^{\rho}$. In this case, all invariant control sets $D$ of $(\mathrm{C})$ are closed and have nonvoid interior, see [7], i.e., we can use the stability concept described in Section 2(I), after Remark 8. The goal is to obtain a complete overview over the stationary solutions of (S) and their stability behavior, depending on the two parameters $\alpha \in \mathbb{R}$ and $\rho \geq 0$. This behavior does not depend explicitly on the vectorfields $X$ and $Y$, but on the bifurcation diagram of the deterministic system

$$
\dot{x}=X(x)+\alpha Y(x), \quad \alpha \in \mathbb{R} .
$$

Therefore, we will use typical (parts of) bifurcation diagrams and discuss their random perturbations.

\subsection{The transcritical bifurcation}

A transcritical bifurcation diagram, where the slopes of the bifurcation branches have the same sign, is shown in Figure 1.

For $\rho=0$, i.e., $\alpha_{1}=\alpha$, we have the deterministic diagram in Figure 1. For $\rho>0$ we visualize the stochastic situation in the following way: We know from Section 2 that stationary solutions can exist only in control sets, and that control sets form around the steady states of (D), i.e., around the branches of a bifurcation diagram. For a given perturbation range $\rho$ we indicate the control sets of the system $\left(\mathrm{C}^{\beta}\right)$ as areas around the branches for all $\alpha$-values. A typical perturbation of Figure 1 is shown in Figure 2. For a fixed $\alpha \leq \alpha_{1}$ there exist two control sets $A(\alpha)$ and $B(\alpha)$, which 


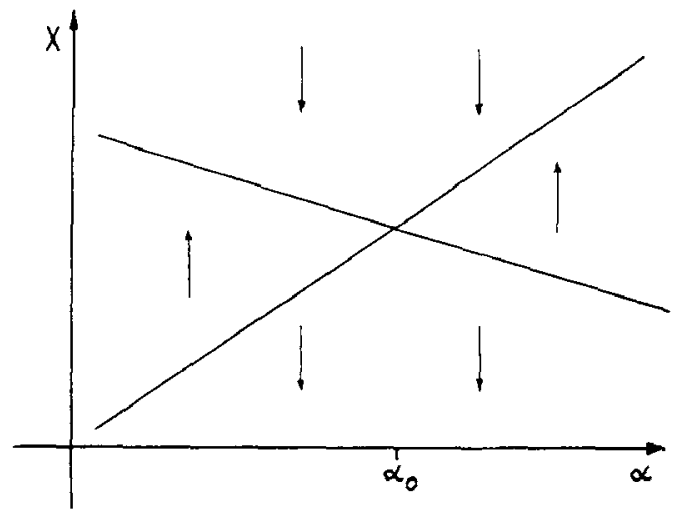

Fig. 6. Transcritical bifurcation with slopes of different sign.

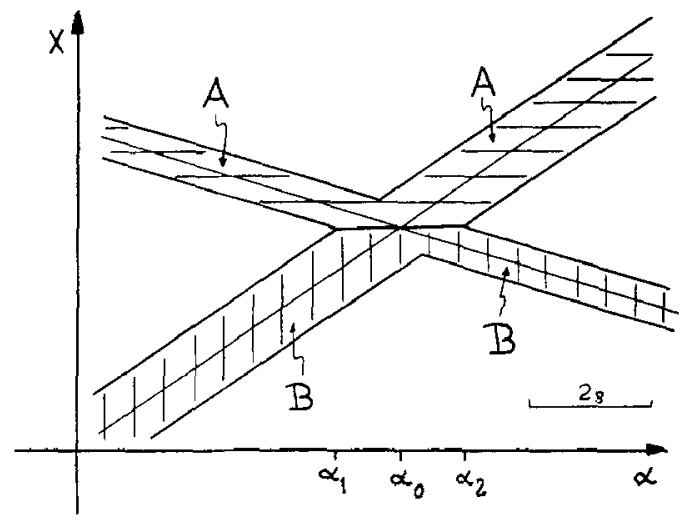

Fig. 7. Random perturbation of size $\rho$ for Figure 6 .

If the arrows in Figure 1 are reversed, the behavior of $(S)$ is similar, except that the system moves upward now.

A transcritical bifurcation, where the slopes of the bifurcation branches have different sign, is presented in Figure 6.

In this case, the random perturbation shows a completely different behavior, see Figure 7.

For all $\alpha \in \mathbb{R}$ there exists a (unique) stationary Markov solution in area $A$, whose support has nonvoid interior. No exchange in stability can be determined. The only specific feature for $\alpha \in\left\{\alpha_{1}, \alpha_{2}\right\}$ is that the bistability area of the Markov solutions in $B$ touches the support of the invariant distribution, i.e., the strict domain of attraction $\tilde{A}(A(\alpha))$ contains only those points $y \in \mathbb{R}$ that lie above the lower boundary of $A(\alpha)$. Of course the domain of attraction $\mathscr{A}(A(\alpha))$ contains also $B(\alpha)$.

Again, reversing the arrows in Fig. 6 leads to a similar behavior of (S), where the stationary Markov solutions are now concentrated around the lower branches.

The separating case between Figures 1 and 6 is the one with a horizontal branch. This case will be discussed in Section 4 .

\subsection{The saddle-node bifurcation}

The typical diagram for a saddle-node bifurcation is shown in Figure 8.

The perturbation diagram in Figure 9 has the following features:

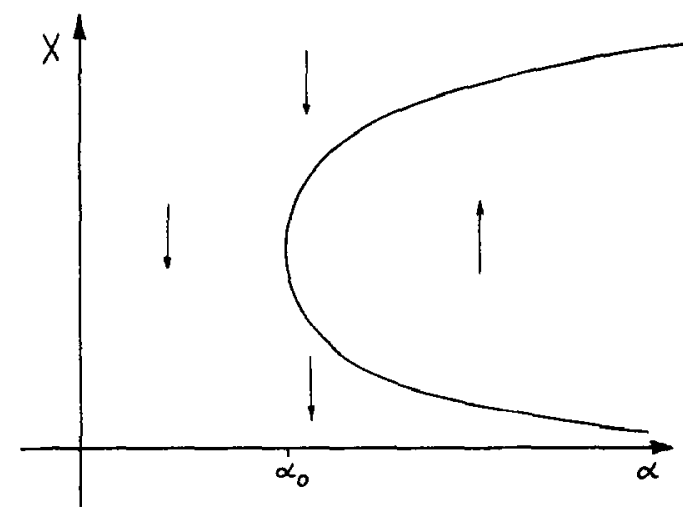

Fig. 8. Diagram of a saddle-node bifurcation.

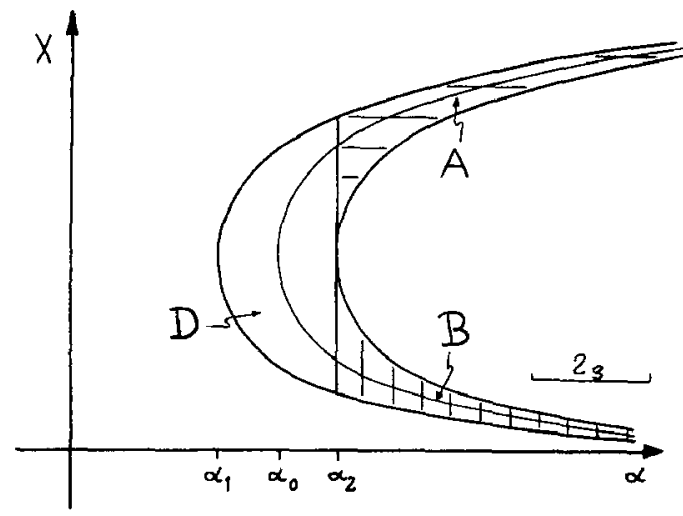

Fig. 9. Perturbation of the saddle-node diagram. 
the exit probability $p(y)$ and of the expected exit time $\tau_{B}(y)$ depends, of course, on the statistics of $\alpha_{t}^{\rho}$.

Hence, for $\alpha \leq \alpha_{1}$ the stochastic system mimics in principle the behavior of the deterministic system (D), except that the stationary solution in $A$ and the bistability region in $B$ occur on sets with nonvoid interior. For increasing $\rho>0$, the control sets become larger, and the point $\alpha_{1}$ decreases.

- For $\alpha \geq \alpha_{2}$, the behavior is similar to the one for $\alpha \leq \alpha_{1}$. The point $\alpha_{2}=\alpha_{0}+\rho$ increases with $\rho$.

- For $\alpha \in\left(\alpha_{1}, \alpha_{2}\right)$, there is no invariant control set, i.e., no stationary Markov solution exists. Region $C$ consists of variant control sets, containing (non unique) stationary flow solutions. Every Markov solution of the stochastic system, starting above the upper boundary of $C(\alpha)$, will cross through $C(\alpha)$ in finite time, even with finite expectation of the passing time. However, the process will slow down in $C(\alpha)$ considerably, due to the existence of a branch of stable fixed points, see Figure 5 for the expected passing time $\sigma_{C}(y)$ through $C(\alpha)$ for initial conditions $y \geq \inf C(\alpha)$.

The exact form of $\sigma_{C}$ depends again on the statistics of $\alpha_{t}^{\rho}$.

This behavior of (S) is completely different from the characteristics of (D). While for (D) only the dynamics at $\alpha \in\left(\alpha_{1}, \alpha_{2}\right)$ play a role, the stochastic system incorporates the entire dynamics in $[\alpha-\rho, \alpha+\rho]$, which leads to this 'break through' effect, due to the presence of unstable fixed points. As $\rho$ increases, the 'break through' gap increases as.

The behavior of a random perturbation of a transcritical bifurcation diagram can be described using the two parameters $\alpha$ and $\rho$ : For increasing $\alpha$ the Markov solutions of (S) change their behavior twice: At $\alpha_{1}=\alpha_{0}-\rho$, a stationary Markov solution disappears and a non-Markovian stationary flow solution appears. For any Markov solution of (S) the area of this non-Markovian flow solution is characterized by a considerable slow down. At $\alpha_{2}=\alpha_{0}+\rho$, a stationary Markov solution reappears.

REMARK. It may be interesting to notice the behavior of the associated control system for $\alpha \in\left(\alpha_{0}-\rho, \alpha_{0}+\rho\right)$ : On the one hand, on an open and dense set of admissible control functions and initial values in $\mathcal{U}^{\rho} \times \mathbb{R}$, the solutions $\varphi(t, x, u)$ of $\left(C^{\rho}\right)$ will leave the area $C(\alpha)$ (see [8]). On the other hand, when restricted to trajectories that stay within $C(\alpha)$, the corresponding control flow is 'chaotic', compare [9] for a precise statement. Thus $C(\alpha)$ can be described as a region of 'transient chaos' for the control system $\left(\mathrm{C}^{p}\right)$.

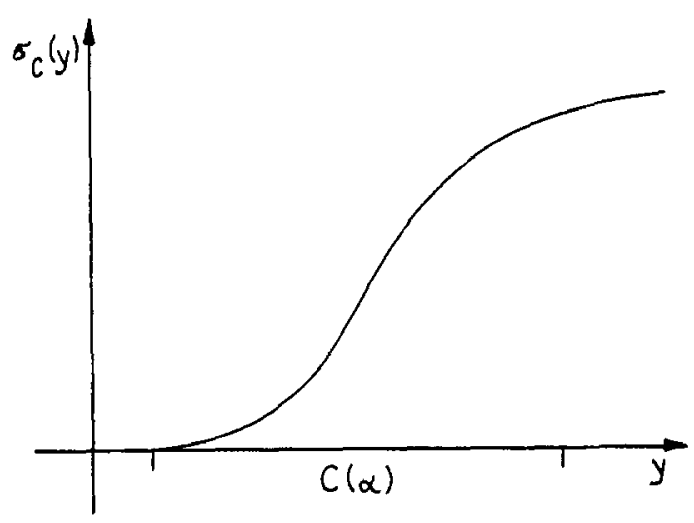

Fig. 5. Expected passing time $\sigma_{C}$. 


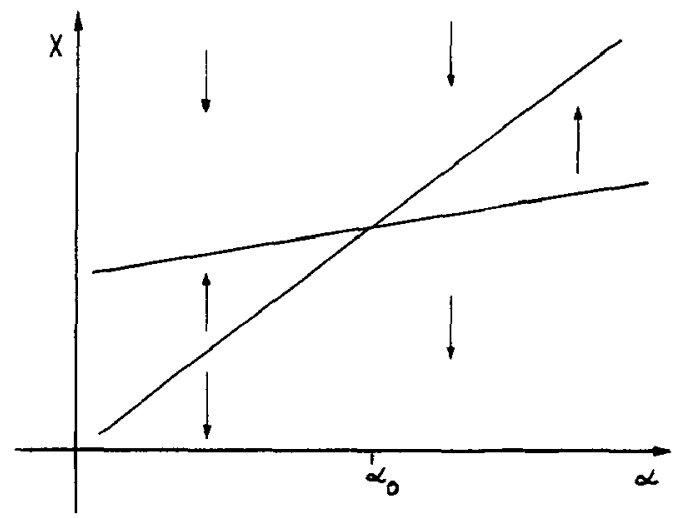

Fig. 1. Transcritical bifurcation with bifurcation point $\alpha_{0}$.

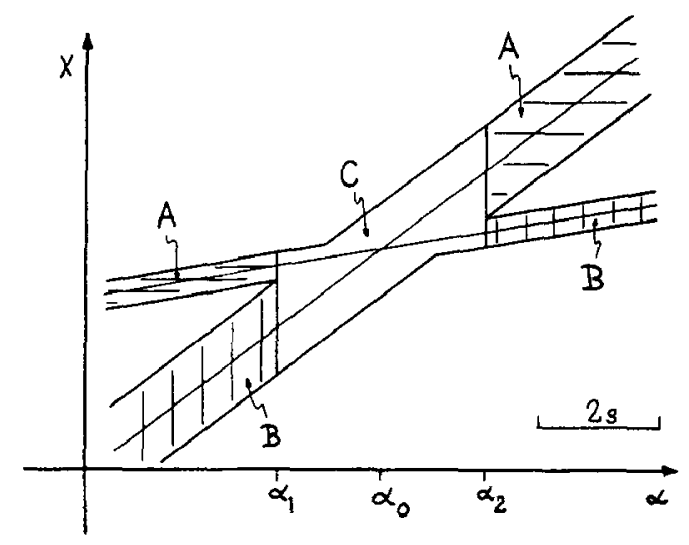

Fig. 2. Random perturbation of size $\rho$ for the bifurcation diagram in Figure 1.

are obtained by projecting the shaded areas labeled $A$ (around the branch of stable steady states) and $B$ (around the unstable steady states) above this $\alpha$-value onto the $x$-axis, and similarly for $\alpha \geq \alpha_{2}$. For $\alpha \in\left(\alpha_{1}, \alpha_{2}\right)$ there exists only one control set $C(\alpha)$, which contains various steady states of the unperturbed system. In general, invariant control sets are shaded horizontally as $A$-areas, variant control sets leading to bistability areas are shaded vertically as $B$-areas, and $C$ and $D$-areas consists of variant control sets, in which the stochastic system exhibits specific behavior without deterministic analogue, such as slowing down or non-existence of any stationary solutions.

For the perturbed system in Figure 2, we obtain the following results, using the theory from Section 2:

- For $\alpha \leq \alpha_{1}=\alpha_{0}-\rho$ there are 2 control sets, those in area $A$ are invariant, those in area $B$ are variant. Hence for each $\alpha \leq \alpha_{1}$, there is a unique stationary, ergodic Markov solution $x_{t}(\alpha)$ with support in $A(\alpha)$, and its stability domain ranges from the upper boundary of $B(\alpha)$ to $\infty$. There also exists a (unique) stationary flow solution in area $B$, but all Markov solutions will leave area $B P^{\rho}$-almost surely, with finite expectation of the exit time. For each initial value $y \in B(\alpha)$ there exists $p(y) \in(0,1)$, such that with probability $p(y)$ the process will leave towards $A$, and with probability $1-p(y)$ it will leave at the lower boundary of $B(\alpha)$, compare Figure 3 . Figure 4 shows the expected exit time $\tau_{B}(y)$ from $B(\alpha)$ for $y \in B(\alpha)$ qualitatively. The exact form of

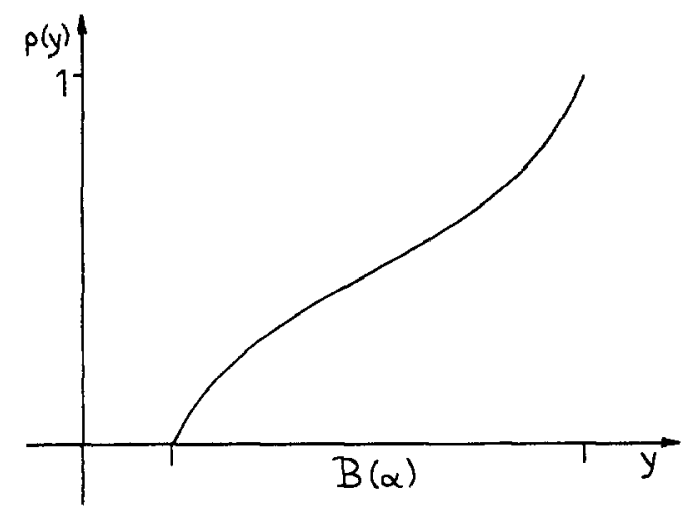

Fig. 3. Exit probability $p(y)$.

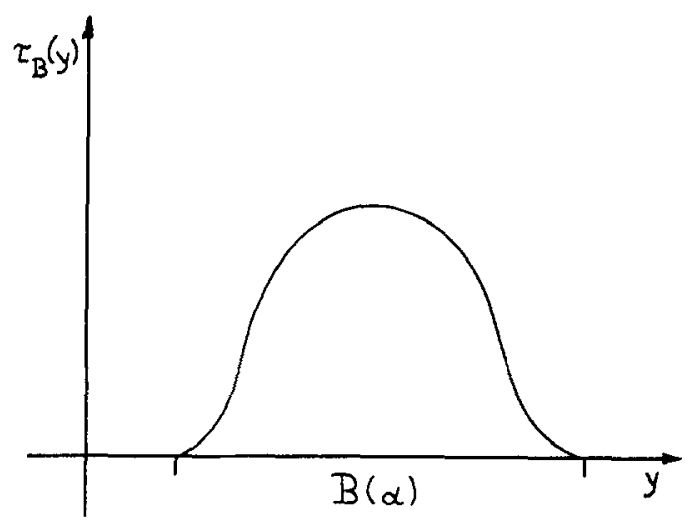

Fig. 4. Expected exit time $\tau_{b}$. 
- $\alpha<\alpha_{1}=\alpha_{0}-\rho$ : No control sets exist.

- $\alpha_{1} \leq \alpha<\alpha_{2}=\alpha_{0}+\rho$ : A variant control set $D(\alpha)$ with stable and unstable fixed points exists. According to Theorem 2.4(1)(iii) no stationary flow solution exists in $D(\alpha)$.

- $\alpha \geq \alpha_{2}$ : In this case, there are two areas of control sets: Area $A$ contains invariant control sets with stationary Markov solutions, area $B$ has variant control sets with stationary flow solutions. The unique Markov solutions are stable within the strict domain of attraction $\mathscr{A}(A(\alpha)$ ), ranging from the upper boundary of $B$ to $\infty . B$ is a bistability region with exit probabilities and expected exit times similar to the ones in Figures 3 and 4. The passing times of the Markov solutions in area $D$ show a behavior similar to the one in Figure 5, although the slowing down effect now depends crucially on $\alpha$ : Slowing down becomes more pronounced, as $\alpha$ increases from $\alpha_{1}$ to $\alpha_{2}$.

If the arrows in Figure 8 are reversed, the behavior of (S) is again similar, with areas $A$ and $B$ interchanged.

REMARK. The perturbation diagrams in Figures 2,7, and 9 describe the possible types of random perturbations of deterministic bifurcation diagrams in the vicinity of bifurcation points for small perturbations, if no common zeros of the right hand side of $(S)$ are present. In the next example, we apply these principles to pitchfork bifurcations and analyze the influence of larger perturbation ranges.

\subsection{The pitchfork bifurcation}

Figure 10 shows a typical pitchfork bifurcation with small (Figure 11) and large perturbation ranges (Figure 12). Note that Figure 11 contains two 'break through' areas with slowing down, with merge into one area of type $C$ in Figure 12, when the perturbation explores more of the global dynamics of the system in Figure 10. This means that we have a 'secondary bifurcation' in the perturbed system for $\rho=\frac{1}{2}\left(\alpha_{1}-\alpha_{0}\right)$. This behavior is another indication that two parameters, $\alpha$ and $\rho$, are necessary to describe the qualitative features of perturbed bifurcation diagrams.

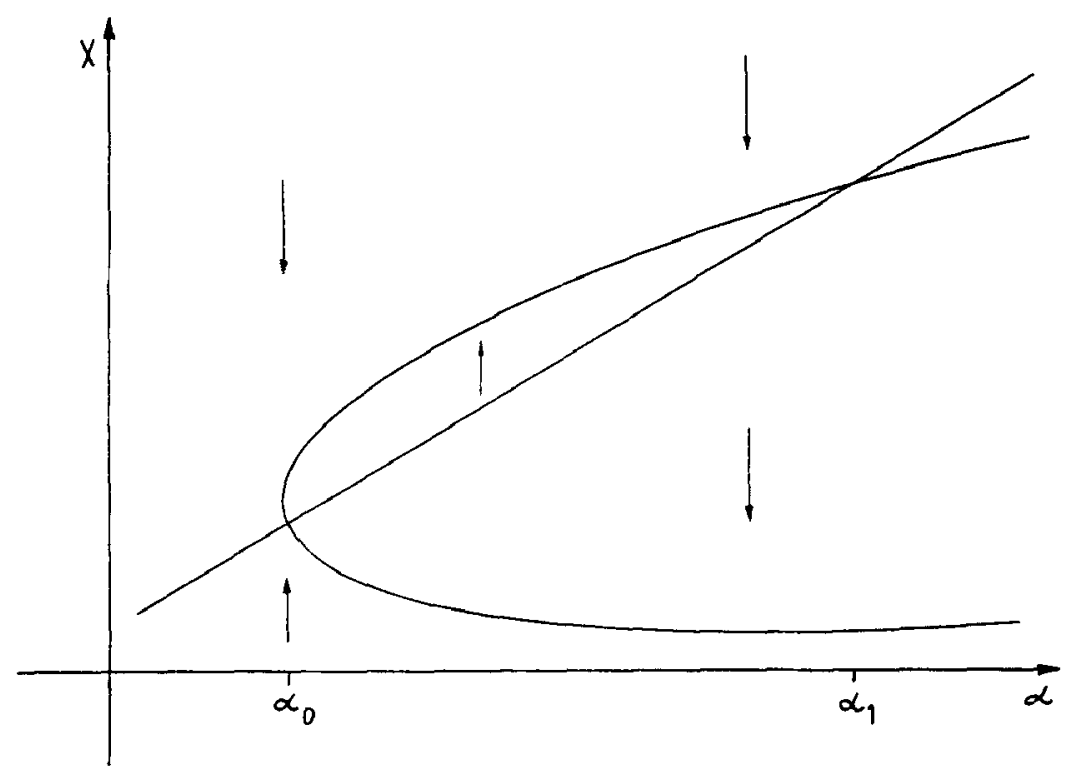

Fig. 10. Diagram of a pitchfork bifurcation. 


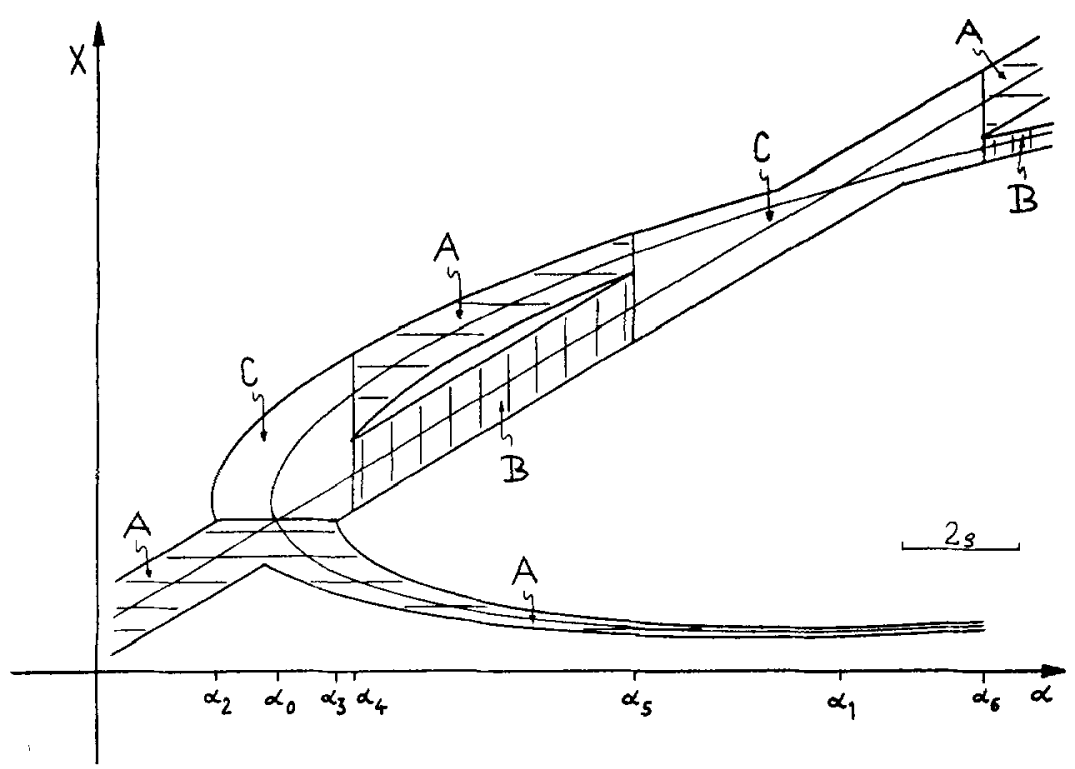

Fig. 11. Perturbation of Figure 10 for small $\rho$.

The perturbation diagrams of other pitchfork type bifurcations can be constructed in a similar manner.

\subsection{Bistability}

Another example that shows how global dynamics can effect perturbed bifurcation diagrams is given by the bistability behavior in an $S$-type bifurcation, see Figures 13-15.

For small perturbation ranges (i.e., $\rho \leq \frac{1}{2}\left(\alpha_{1}-\alpha_{0}\right)$ ) Figure 14 shows a bistability area $B$,

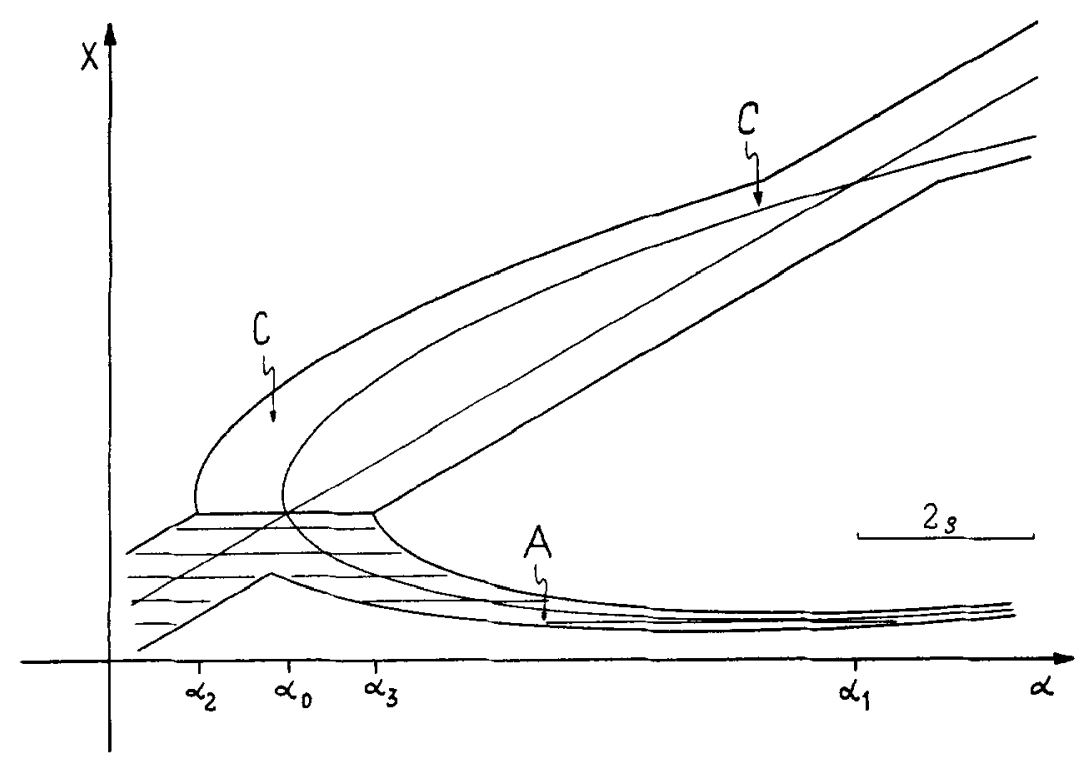

Fig. 12. Perturbation of Figure 10 for large $\rho$. 


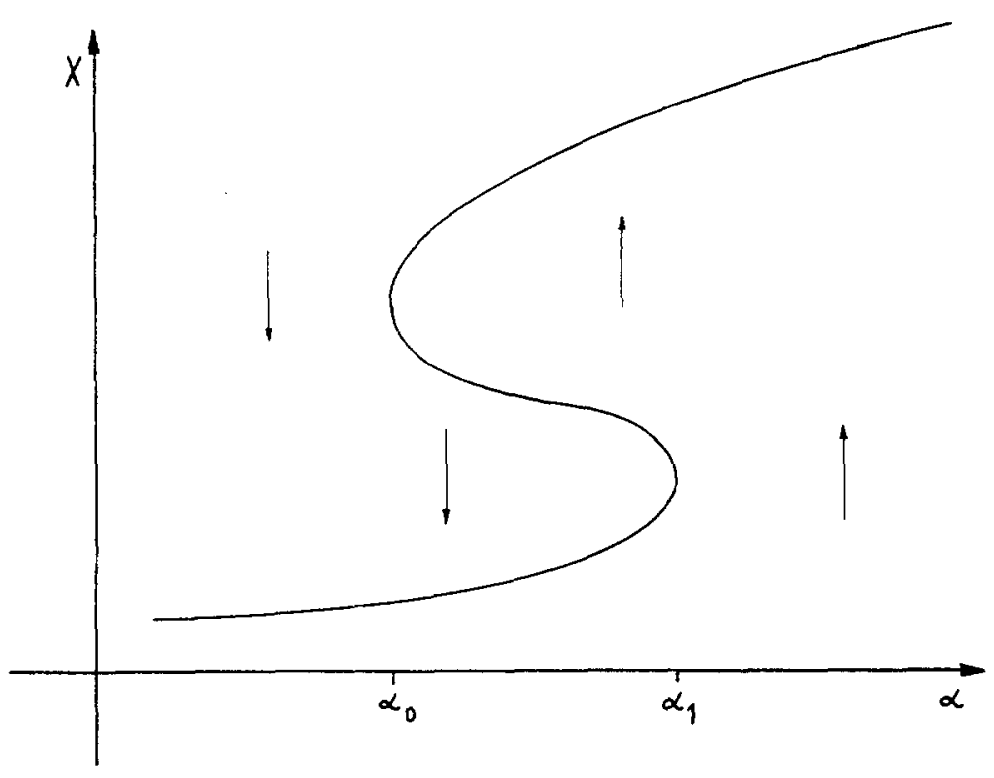

Fig. 13. Diagram of an $S$-bifurcation.

where no stationary Markov, but a stationary flow solution exists. The probability for any Markov solution starting in $B(\alpha)$ to converge towards the invariant Markov distribution in the upper $A$-area is similar to that in Figure $3,1-p(y)$ is the probability of converging towards the lower $A$-area. As $\rho$ increases, the bistability area $B$ becomes smaller and for $\rho>\frac{1}{2}\left(\alpha_{1}-\alpha_{0}\right)$ it is replaced by a region of stationary Markov solutions, whose support now includes the upper and the lower stable branches in Figure 13, as well as the original bistability area. As $\rho$ increases further, the entire bifurcation branches of Figure 13 will lie inside the support of a stationary Markov solution. This example shows that large random perturbations of bistable systems may lead to stable

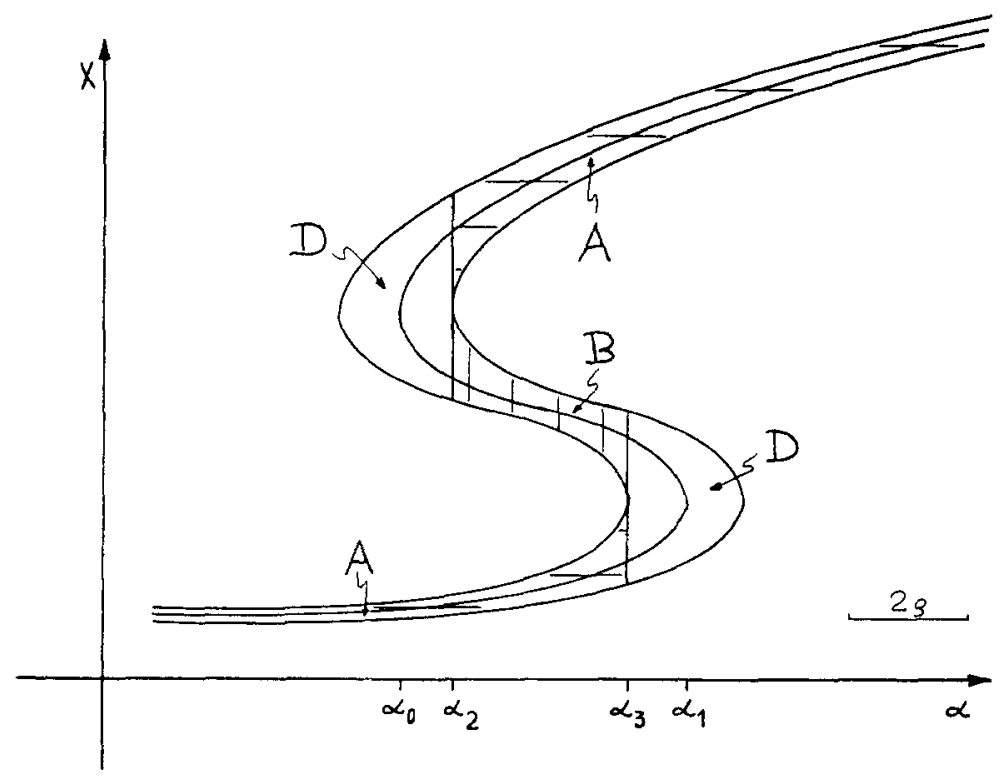

Fig. 14. Perturbation of Figure 13 for small $\rho$. 


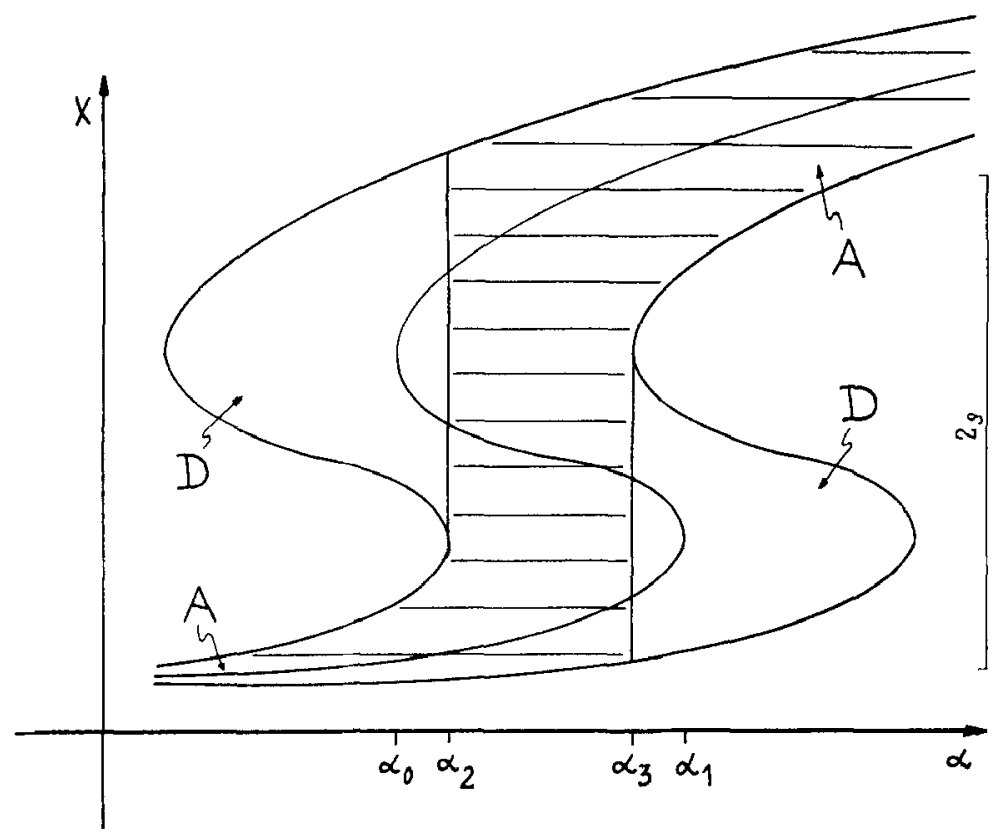

Fig. 15. Perturbation of Figure 13 for large $\rho$.

Markov systems, while for small perturbations the bistability is always maintained, compare Theorem 2.7. The invariant densities associated with Figure 15 will, of course, still show the influence of the original bistability area, see Figure 16. But the precise shape of these densities depends crucially on the statistics of $\alpha_{t}^{\rho}$ and on the vectorfields $X$ and $Y$.

All results obtained in this section are based on the connection of stationary Markov solutions (of stochastic systems with bounded noise) with control systems via the support theorem. The main tool is the global analysis of nonlinear control system, see, e.g. [10] for an overview. This approach does not need the statistics of the noise $\alpha_{t}^{\rho}$ for the description of stationary solutions, their support and stability properties. Of course, the precise form of exit probabilities, passing times and invariant densities do depend on the statistics of $\alpha_{t}^{p}$, and we have sketched the typical behavior of these quantities, since they cannot be computed explicitly, even if the vectorfields

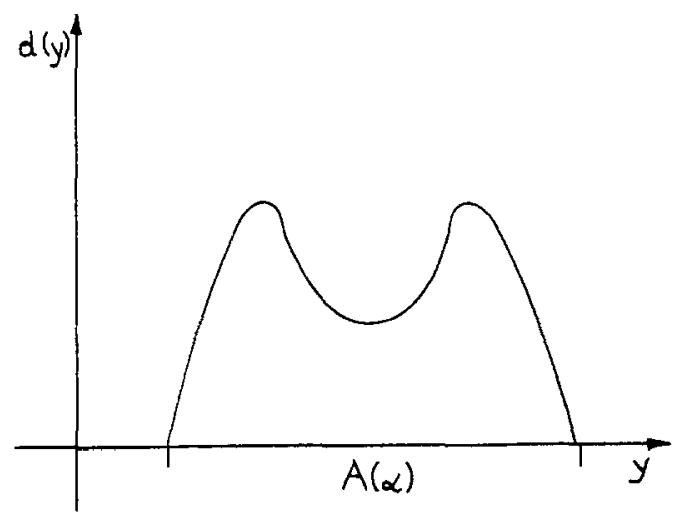

Fig. 16. Typical invariant density for the stationary Markov solution of Figure 15, whose support includes all three bifurcation branches. 
governing the background noise $\beta_{t}$, and the maps $f^{\rho}$ are given. One has to resort to numerical simulations.

The situation is different if we analyze systems with a common fixed point, since the Lyapunov exponent for the fixed point depends on the statistics of $\boldsymbol{\alpha}_{1}^{p}$, compare formula (2.3). These cases are discussed in the next section.

\section{Random Perturbations of Bifurcation Diagrams II: Singular Case}

This section is devoted to perturbations of bifurcation diagrams for systems of the form

$$
\dot{x}=X(x)+\alpha Y(x),
$$

such that there exists $x^{0} \in \mathbb{B}$ with $X\left(x^{0}\right)+\alpha Y\left(x^{0}\right)=0$ for all $\alpha \in A$, with $A$ the considered range of the bifurcation parameter $\alpha$. Without loss of generality we will assume that $A$ is an interval. We consider the stochastic system

$$
\dot{x}=X(x)+\alpha_{t}^{\rho} Y(x), \alpha_{t}^{\rho}(\omega) \in[\alpha-\rho, \alpha+\rho],
$$

such that $\alpha-\rho, \alpha+\rho \in A$.

Obviously, the fixed point $x^{0}$ gives rise to a stationary Markov solution of $\left(\mathrm{S}^{\rho}\right)$, with invariant measure $\delta_{x^{0}}$ in the state component. The stability of this solution cannot be described as in Section 3., since for initial values $y \in \mathbb{R} \backslash\left\{x^{0}\right\}$ the Markov solution $\varphi(t, y, \omega)$ will not enter the support of the invariant measure in finite time. Hence we use the exponential growth behavior of solutions close $x^{0}$ to analyze stability, compare the definition of Lyapunov exponents in Section 2. In order not to introduce a bias through the statistics of $\alpha_{t}^{p}$, we assume:

$$
\mathbb{E} \alpha_{t}^{\rho}=\mathbb{E}_{\eta} f^{\rho}(p)=\alpha .
$$

In this case, comparing the Lyapunov exponents of (D) and $\left(S^{\rho}\right)$ we obtain

$$
\lambda=D_{x} X\left(x^{0}\right)+\alpha D_{x} Y\left(x^{0}\right)=\lambda(\omega) \quad P^{\rho} \text {-almost surely, }
$$

i.e., the steady state solution of (D) and the stationary Markov solution $x^{0}$ of $\left(S^{p}\right)$ change their stability at the same point $\alpha_{0}$ with $\lambda_{\alpha_{0}}=0$.

If $\alpha_{0}$ is a bifurcation point of (D) with respect to $x^{0}$, then there exist additional branches of steady states around $\alpha_{0}$, which may be stable or unstable. This determines the behavior of Markov solutions of $\left(\mathrm{S}^{p}\right)$ around $x^{0}$, as can be seen in the analysis of the following situations.

\subsection{The supercritical bifurcation}

The typical diagram of a supercritical bifurcation with common steady state is given in Figure 17. Concerning the Markov solutions of the corresponding perturbed system we obtain:

- The stationary Markov solution $\left(\beta_{t}, x^{0}\right)$ is exponentially stable for $\alpha<\alpha_{0}$, exponentially unstable for $\alpha>\alpha_{0}$, since the Lyapunov exponent crosses through zero at $\alpha_{0}$.

- There exists a region $B$ with a stationary flow solutions in $\overline{B(\alpha)}$ that are not Markovian. For the Markov solutions this represents a region of bistability for $\alpha<\alpha_{0}$, i.e., these solutions will 


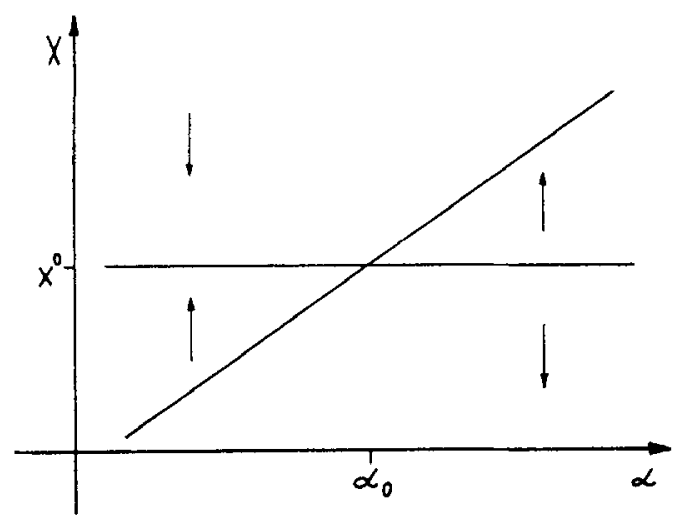

Fig. 17. Supercritical bifurcation with common steady state.

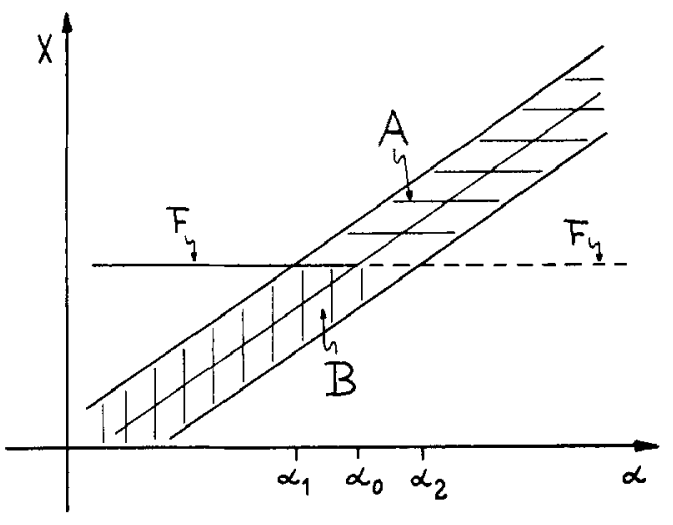

Fig. 18. Random perturbation of the diagram in Figure 17.

converge towards $x^{0}$ with positive probability, and they will leave $B(\alpha)$ at the lower boundary point with positive probability. For $\alpha>\alpha_{0}$, the Markov solutions will leave $\overline{B(\alpha)}$ with probability 1 and decrease thereafter.

- The region $A$ consists of invariant control sets, which are compact for $\alpha>\alpha_{2}$. Hence in this area stationary Markov solutions exist, and they are stable for any Markov solution with initial value $y>x^{0}$. For $\alpha \leq \alpha_{2}$, the invariant control sets $A(\alpha)$ are not compact, hence Theorem 2.3(ii) does not apply. The existence of stationary Markov solutions in this area depends on the dynamics of the systems, i.e., on the vectorfields $X$ and $Y$, and not only on the bifurcation diagram. But we still know that stationary Markov solutions, if they exist in $A(\alpha)$, have support equal to $\overline{A(\alpha)}$. But for $\alpha<\alpha_{0}, x^{0}$ is almost surely exponentially stable, hence there cannot be a stationary Markov solution for $\alpha_{1}<\alpha<\alpha_{0}$ in area $A$, compare Proposition 2.9. On the other hand, for $\alpha \in\left(\alpha_{1}, \alpha_{2}\right], x^{0}$ is almost surely exponentially unstable, and $\max A(\alpha)$ is an entrance boundary. Hence bounded Markov solutions exist in $A(\alpha)$, whose limit sets for $t \rightarrow \infty$ are in $A(\alpha) P^{\rho}$-almost surely. These solutions cover all of $A(\alpha)$, and $A(\alpha)$ is attractive for initial values $y>x^{0}$. However, with the techniques from Section 2 we were not able to show the existence of a stationary Markov solution in $A(\alpha)$ for $\alpha \in\left(\alpha_{0}, \alpha_{2}\right)$.

Similar results hold for supercritical bifurcation diagrams with reversed arrows, and for subcritical bifurcations with common steady state. Note that Figure 17 is the limiting case between Figures 1 and 6 .

\subsection{The perfect pitchfork bifurcation}

Consider the bifurcation diagram in Figure 19 and its perturbation in Figure 20. The analysis in this case is similar to the one for supercritical bifurcations:

- The steady state Markov solution $x^{0}$ loses stability at $\alpha=\alpha_{0}$.

- For $\alpha \in\left[\alpha_{1}, \alpha_{0}\right)$, no stationary Markov solution exists in the region $A$, but any Markov solution with initial value $y \neq x^{0}$, experiences slow down in $A(\alpha)$, and converges towards $x^{0}$ exponentially, locally around $x^{0}$. For $\alpha \in\left(\alpha_{0}, \alpha_{2}\right]$ bounded Markov solutions exist in $A(\alpha)$, with domain of attraction $y>x^{0}$ (and $y<x^{0}$, respectively) for the upper and the lower branch of the region $A$. Finally, for $\alpha>\alpha_{2}$, (unique) stationary Markov solutions exist in $A(\alpha)$, which are globally attractive for $y>x^{0}$, and $y<x^{0}$, respectively. 


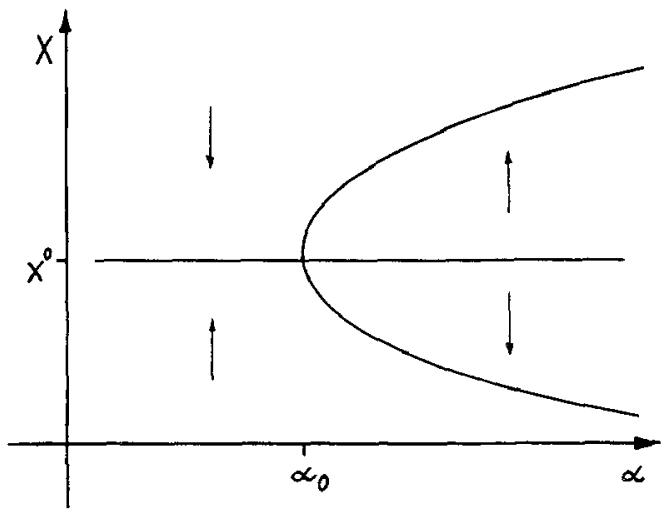

Fig. 19. Diagram of a perfect pitchfork bifurcation.

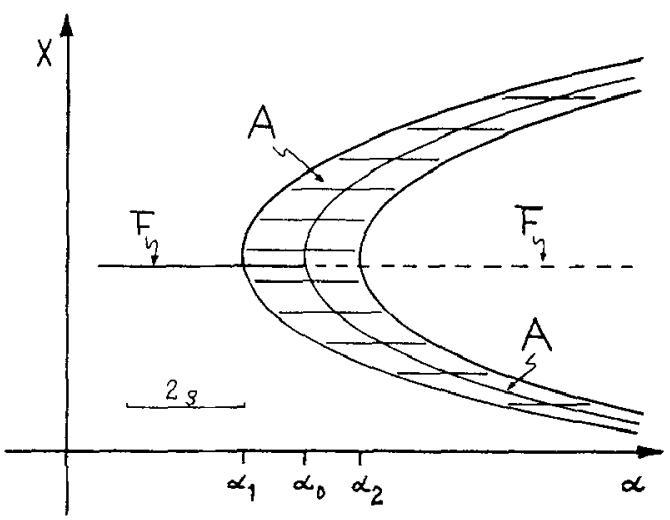

Fig. 20. Perturbation of the diagram in Figure 19.

In comparing Figure 20 with Figure 11 we notice that the slope of the line of steady states in the pitchfork bifurcation plays an important role for the behavior of the random, perturbed system: If the slope of this line is positive (Figures 11 and 12), a system with initial value $y$ above the line, starting with $\alpha<\alpha_{0}$, will follow, for slowly increasing $\alpha$, the stationary solutions in the region $A$ around the lower bifurcation branch. If the slope is $\geq 0$, the system will follow the upper area of region $A$. This effect, which is accompanied by slowing down in certain $\alpha$-intervals, cannot be seen in the deterministic differential equation, and hence can be considered as a 'stochastic symmetry breaking' for diagrams with positive slope of the steady state line.

The behavior discussed for Figures 18 and 20 describes the local analysis around bifurcation points of the deterministic system (D). The next example incorporates some global effects, which may occur for larger perturbation ranges.

\subsection{The imperfect pitchfork bifurcation}

We consider an imperfect pitchfork bifurcation with horizontal steady line as in Figure 21, with small and large perturbations according to Figures 22 and 23.

The small perturbation in Figure 22 exhibits the following kinds of behavior:

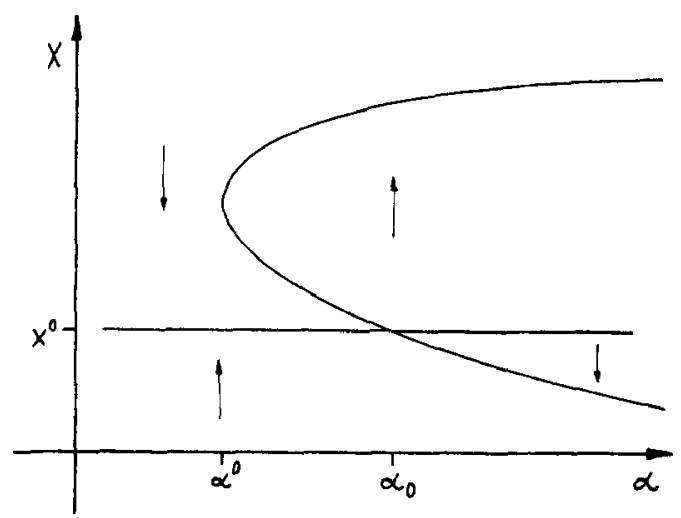

Fig. 21. Example of an imperfect pitchfork bifurcation.

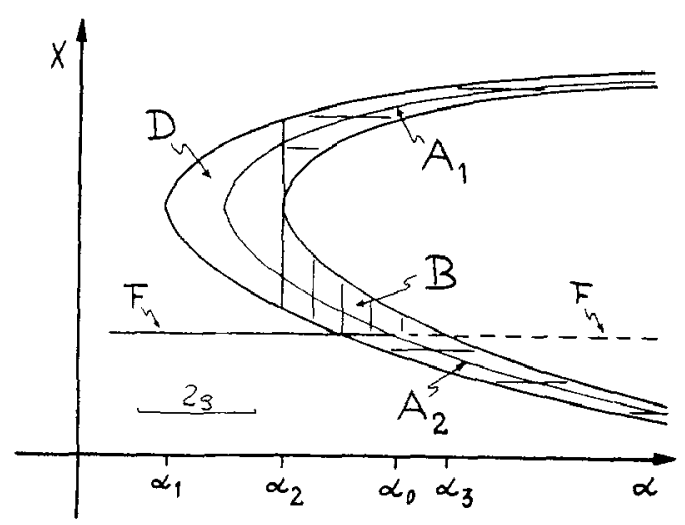

Fig. 22. Perturbation of Figure 21 for small $\rho$. 


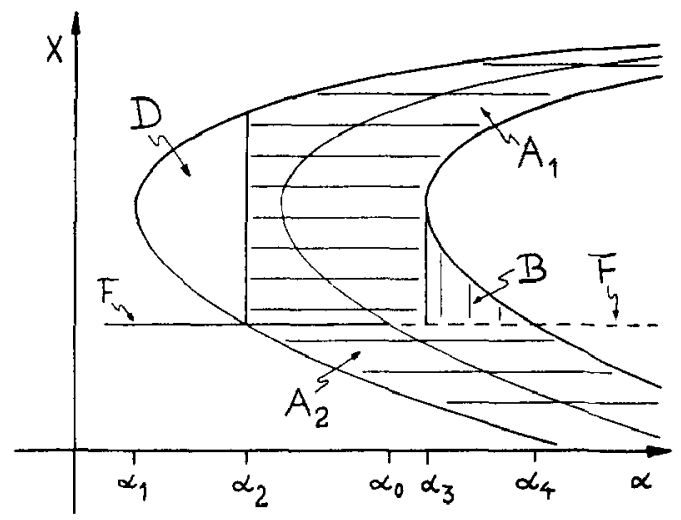

Fig. 23. Perturbation of the diagram in Figure 21 for large $\rho$.

- The steady state Markov solution loses stability at $\alpha=\alpha_{0}$.

- For $\alpha \in\left(\alpha_{1}, \alpha_{2}\right)$, the region $D$ does not support stationary solutions, but Markov solutions experience slow down in this region, compare Figure 9.

- For $\alpha \in\left[\alpha_{2}, \alpha_{0}\right)$, there are unique stationary Markov solutions in region $A_{1}$, and region $B$, consisting of areas of stationary flow solutions in $\overline{B(\alpha)}$, is a bistability region for all Markov solutions with initial values in $B(\alpha)$.

- For $\alpha \in\left(\alpha_{0}, \alpha_{3}\right)$ all Markov solutions with initial value $y>x^{0}$ will converge in distribution towards the invariant measure in $A_{1}(\alpha) . B$ is a region of slowing down for Markov solutions with initial value in $B(\alpha)$.

- For $\alpha \geq \alpha_{3}$ all Markov solutions with initial value $y>x^{0}$ converge to $A_{1}(\alpha)$, without effects of slowing down.

- The behavior of solutions with initial value $y<x^{0}$ is similar to the one described for region $A$ in Figure 18.

For large $\rho$, i.e., $\rho \geq \alpha_{0}-\alpha^{0}$, the perturbation of the imperfect pitchfork bifurcation diagram shows a somewhat different behavior, see Figure 23.

- The bistability area in region $B$, which was found in Figure 22, has disappeared, and for $\alpha \in\left(\alpha_{2}, \alpha_{0}\right)$ the region $A_{1}$, consisting of invariant control sets, is attracted to stable Markov steady state $x^{0}$. We have exponential convergence towards $x^{0}$ in a neighborhood (depending on the trajectory $\omega)$ around $x^{0}$, and slow down in $A_{1}(\alpha)$. For $\alpha \in\left(\alpha_{0}, \alpha_{3}\right), x^{0}$ is unstable, so the region $A_{1}(\alpha)$ contains bounded Markov solutions that are attractive for initial values $y>x^{0}$.

- For $\alpha \in\left[\alpha_{3}, \alpha_{4}\right)$, region $B$ leads to slowing down of the Markov solutions that start in $B(\alpha)$. But the stationary Markov solution in $A_{1}(\alpha)$ is globally attractive for all Markov solutions with initial value $y>x^{0}$.

Note that the bistability area in region $B$ exists for $\rho<\alpha_{0}-\alpha^{0}$, i.e., its existence depends on both bifurcations points of the deterministic diagram and on the perturbation range $\hat{\rho}$, and therefore is a phenomenon depending on the global dynamics.

\section{References}

1. Arnold, L. and Boxler, P., 'Stochastic bifurcation: instructive examples in dimension one', Diffusion Processes and Related Problems in Analysis, Vol. II, Pinsky, M. and Wihstutz, V., eds., Birkhäuser, 1991.

2. Arnold, L. and Kliemann. W., 'On unique ergodicity for degenerate diffusions', Stochastics 21, 1987, 41-61. 
3. Baxendale, P., 'Invariant measures for nonlinear stochastic differential equations', Lyapunov Exponents, Arnold, L., Crauel, H., and Eckmann, J.-P., eds., Lecture Notes in Mathematics 1487, Springer-Verlag, 1991, 123-140.

4. Benoit, E., ed., Dynamic Bifurcations, Lecture Notes in Mathematics 1493, Springer-Verlag, 1991.

5. Boxler, P., 'A stochastic version of center manifold theory', Probability Theory and Related Fields 83, 1989, 509-545.

6. Colonius, F., Häckl, G., and Kliemann, W. 'Controllability near a Hopf bifurcation', Proceedings of the 31 st IEEE Conference on Decision and Control, 1992.

7. Colonius, F. and Kliemann, W., 'Remarks on ergodic theory of stochastic flows and control flows', Diffusion Processes and Related Problems in Analysis, Vol. II, Pinsky, M. and Wihstutz, V., eds., Birkhäuser, 1991, $203-240$.

8. Colonius, F. and Kliemann, W.. 'Limit behavior and genericity for nonlinear control systems', J. Diff. Equations, 1993, to appear.

9. Colonius, F, and Kliemann, W., 'Some aspects of control systems as dynamical system', J. Dynamics Diff. Equations, 5, 1993, 469-494.

10. Colonius, F. and Kliemann, W., Control theory and dynamical systems, Schwerpunktprogramm der DFG 'Anwendungsbezogene Optimierung und Steuerung', report no. 328. Universität Augsburg, Augsburg FRG, 1991.

11. Crauel, H., Markov Measures for Random Dynamical Systems, preprint. Universität Bremen, Bremen FRG, 1990.

12. Gihman, I. I. and Skorohod, A. V., The Theory of Stochastic Processes III, Springer-Verlag, 1979.

13. Guckenheimer, J. and Holmes, P., Nonlinear Oscillations, Dynamical Systems and Bifurcation of Vectorfields (2nd ed.), Springer-Verlag. Berlin, 1986.

14. Hörmander, L., 'Hypoelliptic second order differential equations', Acta Mathematica 119, 1967, $147-171$.

15. Horsthemke, W. and Lefever, R., Noise-Induced Transitions, Springer-Verlag, Berlin, 1980.

16. Iooss, G, and Joseph, D.D. Elementary Stability and Bifurcation Theory, Springer-Verlag, Berlin, 1980.

17. Kifer, Y., Random Perturbations of Dynamical Systems, Birkhäuser, Basel, 1988

18. Kliemann, W., 'Recurrence and invariant measures for degenerate diffusions', Annals of Probability 15, 1987, $690-707$.

19. Kunita, H., Diffusion Processes and Control Systems, Lecture Notes, University of Paris, 1974.

20. Kunita, H., 'Supports of diffusion processes', Proceedings International Symposium on Stochastic Differential Equations, Ito, K., ed., Wiley, 1978, 163-185.

21. Rozanov, Y. A. Stationary Random Processes, Holden-Day, 1967.

22. Ruelle, D., Elements of Differentiable Dynamics and Bifurcation Theory, Academic Press, New York, 1989.

23. Stroock, D. W. and Varadhan, S. R. S., On the support of diffusion processes with applications to the strong maximum principle', Proceedings of the 6th Berkeley Symposium on Mathematical Statistics and Probability 3, 1972, $333-359$.

24. Ventcel, A. D. and Freidlin, M.I., Random Perturbations of Dynamical Systems, Springer-Verlag, Berlin, 1984.

25. Zeeman, E. C., 'Stability of dynamical systems', Nonlinearity 1, 1988, 115-155. 